\title{
Fenomenología
}

\section{El estudio de la fenomenología como una vía de acceso a la mejora de los cuidados de enfermería.}

\section{The study of the phenomenology as one way of access for the improvement of the nursing assistance.}

\section{Estudo da fenomenologia como alternativa de melhoria aos cuidados de enfermagem.}

\author{
${ }^{1}$ Patrícia Campos Pavan Baptista - ${ }^{2}$ Miriam Aparecida Barbosa Merighi - ${ }^{3}$ Genival Fernandes de Freitas \\ ${ }^{1}$ Professora Doutora do Departamento de Orientação Profissional. \\ ${ }^{2}$ Professora Titular do Departamento de Enfermagem Pediátrica. \\ ${ }^{3}$ Professor Doutor do Departamento de Orientação Profissional. \\ Escola de Enfermagem da Universidade de São Paulo.
}

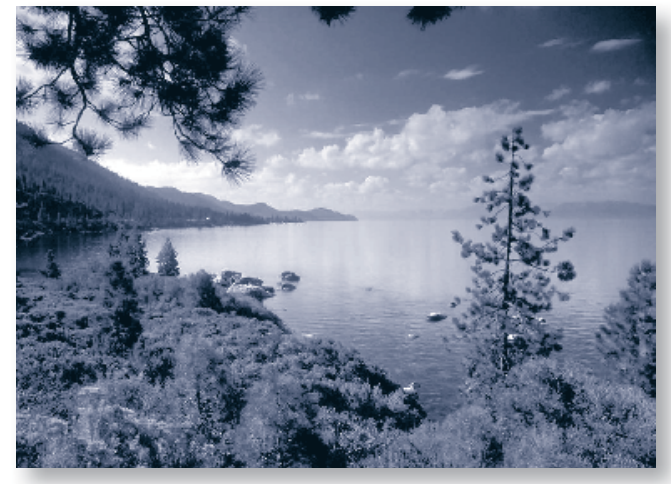

The study of the phenomenology as one way of access for the improvement of the nursing assistance

\section{ABSTRACT}

In the nursing, an increasing search for the research of qualitative nature, caused for the desire of the researchers in knowing the experiences of an illness not only is observed or determined events under the approach of the citizen and under the pathophysiological aspects. The present article is about an update study, in which if it intends to rescue the relevance of the study of the phenomenology for improvement in the quality of the assistance of nursing given to the customers, in view of whom our objective profession the satisfaction of the necessities basic human beings as well as the promotion of a care that exceeds the illness, and can articulate it the existence of the citizens.

Key words: qualitative research, phenomenology, nursing

Estudo da fenomenologia como alternativa de melhoria aos cuidados de enfermagem

\section{RESUMO}

$\mathrm{Na}$ enfermagem, observa-se uma busca crescente pelas pesquisas de natureza qualitativa, ocasionada pelo desejo dos pesquisadores em conhecer as experiências de uma doença ou determinados eventos sob o enfoque do sujeito e não apenas sob os aspectos fisiopatológicos. O presente artigo trata-se de um estudo 
de atualização, no qual se pretende resgatar a relevância do estudo da fenomenologia para melhoria na qualidade da assistência de enfermagem prestada aos clientes, tendo em vista que a nossa profissão objetiva a satisfação das necessidades humanas básicas assim como a promoção de um cuidado que transcenda a doença, e possa articular-se à existência dos sujeitos.

Palavras-chave: pesquisa qualitativa, fenomenologia, enfermagem

\section{El estudio de la fenomenología como una vía de acceso a la mejora de los cuidados de en- fermería}

\section{RESUMEN}

En la enfermería, se observa una búsqueda creciente por las investigaciones de naturaleza cualitativa, causada por el deseo de los investigadores en conocer las experiencias de una enfermedad o determinados acontecimientos bajo el enfoque del sujeto y no sólo los aspectos fisiológicos y patológicos. El presente artículo trata de un estudio de actualización, en el cual se busca rescatar la importancia del estudio de la fenomenología para la mejoría en la calidad del cuidado de enfermería fortaleciendo a los clientes, teniendo en cuenta que nuestra profesión objetiva la satisfacción de las necesidades humanas básicas así como la promoción de un cuidado que trascienda la enfermedad y pueda articularse a la existencia de los sujetos.

Palabras-clave: investigación cualitativa, fenomenología, enfermería.

\section{INTRODUCCIÓN}

La ciencia moderna ha transformado la sociedad y su desarrollo económico, político y cultural, lo que refleja la vida cotidiana de las personas, valores y creencias. Derivados de las innovaciones tecnológicas, las necesidades humanas han cambiado y la asistencia del hombre contemporáneo requiere una mirada que va más allá del funcionamiento de los sistemas biológicos, llegando a su complejidad y totalidad.

La comprensión de la experiencia del sujeto acerca del proceso de salud y enfermedad consiste en identificar las redes de significados culturales atribuidos a la salud, tratamientos, diagnósticos y sus respectivas acciones (RIVERA, 2006).

Nuevos investigadores están entrando en el campo de la investigación cualitativa, resaltando la necesidad de profundizar los estudios a partir de la singularidad de la experiencia humana. En enfermería, hay una creciente búsqueda de la investigación cualitativa, por el deseo de los investigadores en comprender las experiencias de una enfermedad o determinados acontecimientos desde la perspectiva del sujeto y no en los aspectos fisiológicos y patológicos.

En virtud de este nuevo paradigma, la experiencia de los investigadores en el desarrollo de estudios fenomenológicos ha demostrado ser una puerta para la reflexión y la consecución de la calidad en el proceso de cuidado en enfermería. Hemos observado que al buscar la conciencia y los significados a través de la investigación fenomenológica, las necesidades y potencialidades de los individuos se dan a conocer mejor, y se abren vías importantes para la realización de cambios en los procesos de cuidados de enfermería.

Al volver a la comprensión e interpretación de los fenómenos de investigación, recabando las vivencias de los sujetos sobre sus condiciones en el proceso de salud-enfermedad, posibilita al enfermero aproximarse más a las 


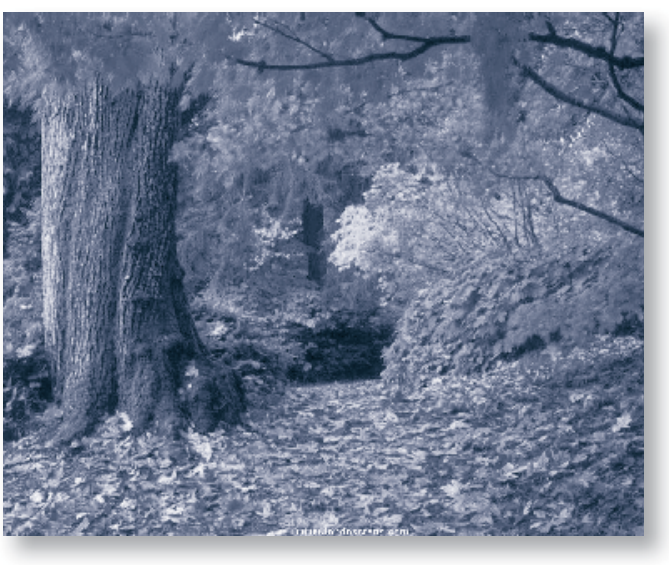

investigaciones de los cuidados de enfermería, creando nuevas posibilidades para una asistencia integral y más humanizada (JESUS, PEIXOTO, CUNHA, 1998).

Por consiguiente, este artículo presenta una actualización en el cual se desea recuperar la relevancia del estudio de la fenomenología para mejorar la calidad de los cuidados de enfermería a los clientes, considerando que nuestra profesión tiene como objetivo satisfacer las necesidades humanas básicas y la promoción de la atención que trascienda la enfermedad, y puedan articularse a la existencia de los sujetos.

La investigación cualitativa fenomenológica: revisando los conceptos

La investigación fenomenológica surgió de una reflexión sobre la crisis de las ciencias, apareciendo un nuevo método de conocimiento y poco a poco ganando reconocimiento como un abordaje a la investigación cualitativa aplicable a los estudios de fenómenos importantes en la vida cotidiana en varias áreas (DARTIGUES, 2003; MOREIRA, 2002).

La fenomenología pasa a ser ampliamente conocida a partir del movimiento filosófico fundado por Edmund Husserl en los albores del siglo XX. En su esfuerzo por consolidar la fenomenología como un movimiento filosófico riguroso, el filósofo trató de contrarrestar el empirismo y el psicologismo, con el objetivo de superar la oposición tradicional entre el realismo y el idealismo. Para Husserl, la fenomenología se fundamenta en la experiencia vivida, posibilitando la mirada de las cosas tal como ellas se manifiestan; describiendo el fenómeno sin explicarlo, sin analizarlo y no importando la búsqueda de relaciones causales (TERRAL ET AL, 2006).

Así, la fenomenología es el estudio de las esencias y se caracteriza por un método de investigación que se ocupa de los fenómenos, o sea, todo lo que se manifiesta, abandonando los preconceptos y presupuestos. La investigación fenomenológica permite al investigador el acceso a la conciencia humana, el regreso "a las cosas mismas". De ese modo, se trata de un método adecuado de estudios en enfermería, pues nos invita a entender el sentido de ser humano en el mundo a partir de su "facticidad" (DARTIGUES, 2003; MERIGHI, 2003).

Es un método de estudio que trata de discutir sobre los nuevos requisitos, las condiciones de la ciencia, tomando como punto de partida los datos inmediatos de la conciencia, la raíz de que se alimenta. Por esa razón, este método se dirige al el interrogativo y al fenómeno que es algo inacabado, donde se puede desvelar el mundo cotidiano del Ser, donde la experiencia se convierte en la descripción de sus vivencias (SILVA, LOPES, DINIZ, 2008).

El punto de partida es "volver a las cosas mismas, reconociendo la prioridad de la práctica, el ámbito de las decisiones y actuar sobre el pensamiento y la reflexión. La experiencia no es sólo la capacidad humana para representar el mundo a través de los procesos cognitivos, ya que se tiene en si misma "significaciones" o "unidades ideales significativas." Para la fenomenología, la operación primordial de significación es que no existe separadamente 
de la expresión y, como tal, es un fenómeno sensible al cuerpo (y no una mera cuestión de la "subjetividad"). En este sentido, el método fenomenológico está dirigido a fijarse en los significados que se dan, y que son tal como son propuestos por nuestras experiencias (ALVES, 2006).

El estudio de la fenomenología exige del investigador una actitud fenomenológica, haciendo hincapié en la utilidad de auto-conocimiento y la transformación de su visión del mundo de los fenómenos vividos en las experiencias cotidianas que se obtienen. Para la investigación fenomenológica es necesario que el investigador use "los ojos" de este enfoque para "ver el sujeto y las cosas del mundo", permitiendo la manifestación natural de las experiencias.

Basándose en esta proposición, el investigador tiene la intención de aprender de aquellos que han vivido o viven una cierta experiencia, considerando que el sujeto que experimenta algo es quien más puede relatar tal vivencia. Así, en la fenomenología, la búsqueda de significados es una tarea fundamental para el investigador que se basa en un método que se presta a llegar una comprensión de los múltiples significados de la experiencia vivida, y que, a su vez, tiene los múltiples contornos. La búsqueda de un sentido en la experiencia mundana incluye una visión del ser humano en mutua constitución con el mundo y con la su historia en el mundo (MOREIRA, 2002).

Aunque reconocida como un método de estudio, la fenomenología sufrió muchas críticas en el pasado, en términos de procedimientos y del punto de vista ético. Las antiguas críticas a las investigaciones cualitativas tuvieron estrecha relación con la baja expresividad numérica de sujetos de la investigación y la falta de claridad en los procedimientos meto- dológicos. En este sentido, en la actualidad se observa mayor atención con el rigor de la descripción metodológica en las investigaciones cualitativas, garantizando la credibilidad a los resultados de las investigaciones que buscan la subjetividad de las experiencias.

En cuanto a la preocupación por el rigor del método en la investigación fenomenológica, estudios más recientes han buscado una mayor integración del objeto investigado con el marco filosófico, proporcionando una mayor credibilidad en la presentación de los fenómenos estudiados (LEITE, 2006; GONÇALVES, 2005).

Mediante el uso de la fenomenología como un método de investigación es necesario un estudio detallado de los diversos filósofos fenomenólogos para elegir el marco que adecue mejor con el objeto investigado y su relación con el sujeto, actor principal de esa experiencia. De esa manera, para comprender los fenómenos de la vida cotidiana se debe hacer un análisis que lleva en consideración un marco filosófico determinado, que corresponde a la experiencia del sujeto en el mundo, en el contexto de las relaciones con el mundo.

Creemos que los avances en los procedimientos metodológicos en la investigación fenomenológica, aquí descritos, representan no sólo un cambio de paradigmas que dificultaran las innovaciones y la profundización en el estudio fenomenológico, como también ellos representan la posibilidad de que los re-

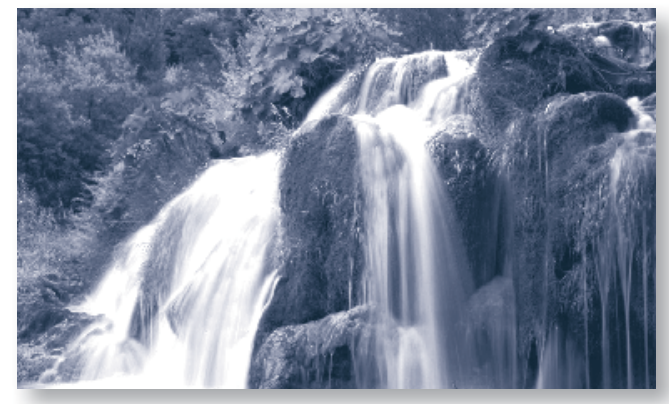


sultados obtenidos con este enfoque sean datos confiables y generadores de impacto para la ciencia en el abordaje sobre el ser humano.

La fenomenología y la práctica de enfermería: reflexiones sobre el cuidar a través de la comprensión de los fenómenos

En el campo de la salud, todavía, domina la influencia del positivismo, que se expresa por la visón centrada en la enfermedad, en la fragmentación del sujeto, reduciendo el ser humano al aspecto biológico. A su vez, la actuación de los profesionales es cientificista y objetivista, intentando adaptar el sujeto a un nivel ideal bienestar (GOMES, PAIVA, VALDES, 2008).

La ruptura de estos paradigmas es una tarea difícil, pero necesaria para transformar la calidad de la atención en salud. El abandono de la fragmentación, la visión reduccionista de la enfermedad y los rituales tan valorados requiere mucho más que un conocimiento metodológico, sino la capacidad de traducir las investigaciones y volverlas en facticidad.

Estudios sobre la experiencia de la enfermedad toman como punto de análisis el presupuesto de que lo social es originariamente toda la situación vivida por los actores sociales en determinados contextos históricos y culturales concretos. El "mundo de la vida" ("Lebenswelt") es el horizonte de referencia paradigmático por el cual los individuos y grupos sociales interactúan, definen acciones previstas de sentido y proporcionan cualquier intervención cognitiva, tales como las "representaciones". El encuentro con el otro no es, por tanto, una realidad contingente de la acción humana, pero el campo mismo de lo que es posible emplear esfuerzos para intervenir en la realidad (ALVES, 2008).

En la fenomenología, más importante es el reconocimiento de la experiencia, porque a medida que compartimos una vivencia, una historia de vida, podemos descubrir los significados ocultos y los fenómenos por las apariencias y, por tanto, es posible llegar a las esencias originarias de los seres humanos.

Ser auténtica es una manera de ser que permite a una persona encontrarse a sí misma, mostrando el fenómeno más original de la verdad, donde la presencia es esencialmente un mundo compartido y desde cualquier lugar del mundo se revelan los fenómenos (HEIDEGGER, 2004).

En el contexto de salud, al tratar de comprender el significado de la experiencia vivida por seres humanos, contribuciones valiosas al conocimiento emergen para el conocimiento de las múltiples dimensiones involucradas en el cuidado de la vida humana, hasta ahora inexploradas (TERRAL et al, 2006).

Por tanto, la comprensión del proceso salud-enfermedad desde el individuo y el grupo social, bien como las experiencias de los procesos fisiopatológicos como fenómenos únicos exigen especificidades diferentes del cuidado. El enfermero debe conocer, identificar y tomar decisiones fundamentadas, buscando una atención integral e individualizada que valore los significados más íntimos de los clientes acerca de la enfermedad y de los tratamientos.

Por lo tanto, también es importante reconocer que la mala salud no siempre está relacionada con la enfermedad, lesión o discapacidad, ya que puede ser un problema existencial y el sufrimiento puede ser expresado en diferentes formas, en contraposición a los patrones establecidos para el cuidado de la salud. Al investigar los fenómenos surgen posibilidades de cambios en los puntos de vista sobre la visión del hombre, la práctica de la salud, el significado del proceso salud-enfermedad, dando lugar a un nuevo conocimiento para la salud (GOMES, PAIVA, VALDES, 2008). 
Este nuevo conocimiento se refleja en la calidad de la atención que ofrecemos a nuestros pacientes, una vez que aprendemos a "ver" las personas más allá de la enfermedad, además de los síntomas y signos, permitiendo que sus necesidades reales sean reveladas $y$, por tanto, posibles de satisfacción. Una comprensión fenomenológica, que busca el ser en la enfermedad y su más rica experiencia permite el diseño de las acciones que están destinadas al paciente y no a la enfermedad misma (FREITAS, MERIGHI, FERNANDES, 2007). Ver dicionário detalhado Público.es Nord-Cinema Sued deutsche.de The White House El Confidencial Marmiton.orgTelegraph.co.uk Vogue Nouvel Obs Zamalek Fans Los Angeles Times

En salud se podría hablar de dos tipos de saberes que por supuesto guardan pluralidades, un saber que emerge de los profesionales de salud con sus propios valores, formas de producción y reproducción del saber, con una forma particular de ver la salud, la enfermedad y la muerte pero en el cual no voy a enfocar el análisis y un saber proveniente del mundo no profesional, de la cotidianidad de las personas, del saber-hacer, con alto contenido simbólico y reproducido por la oralidad. En el saber del mundo no profesional, circulan creencias, ideas, valores sobre lo que significa estar saludable, enfermo, con discapacidad, las formas como se pueden enfrentar esas situaciones no siempre compatibles, comparables o intercambiables con el saber profesional (LOPES, 2010).

La incorporación de nuevos modelos de atención de salud se desprende de las experiencias y percepciones descriptas con eses estudios fenomenológicos, donde el investigador enfermero ha puesto en el contexto estudiado para entender los significados reales vividos. En este sentido, hay que desprenderse de los prejuicios y las etiquetas que a veces nos limi- tan para usar la creatividad a través de la sensibilidad, en la dirección a la práctica de enfermera del cuidado.

\section{CONSIDERACIONES FINALES}

Nuestro caminar en la investigación fenomenológica ha puesto de manifiesto una gran variedad de sentimientos, porque nos damos cuenta de que cada persona enfrenta la enfermedad a su modo, presentando diferentes necesidades de atención o cuidados. El enfermero debe tener en su capacitación académica, los conocimientos teóricos y prácticos para satisfacer las necesidades expresadas por los pacientes que experimentan la enfermedad, sin embargo, no las necesidades no manifestadas requieren una atención que va más allá de los patrones ya establecidos.

Observamos que los pacientes son seres únicos, algunos tardan más tiempo en darse cuenta de sus enfermedades; otros buscan tratamientos y hacen inversiones en sí mismos sin descanso. Esos resultados iluminan nuestra práctica y reducen la posibilidad de alejarse de la esencia humana y de nuestro objeto real de trabajo: el cuidado dispensado a los seres humanos.

Aprendemos con la fenomenología que las historias y los relatos contados por las personas enriquecen nuestro conocimiento para la investigación y para la mejoría de la calidad de los cuidados de enfermería. Considerando que el ser humano sólo existe en la verdad, sólo cuando nos fijamos en los pacientes y en su propia existencia, es que conseguimos entrar en el mundo vivido por ellos y podemos compartir sus sentimientos, dolores, angustias $\mathrm{y}$ ansiedades. Acercarse a la otra persona en su esencia puede representar la oportunidad que el otro sea él mismo auténticamente, y seguir viviendo por los caminos que revelan una exis- 


\section{Cultura de las Cuidados}

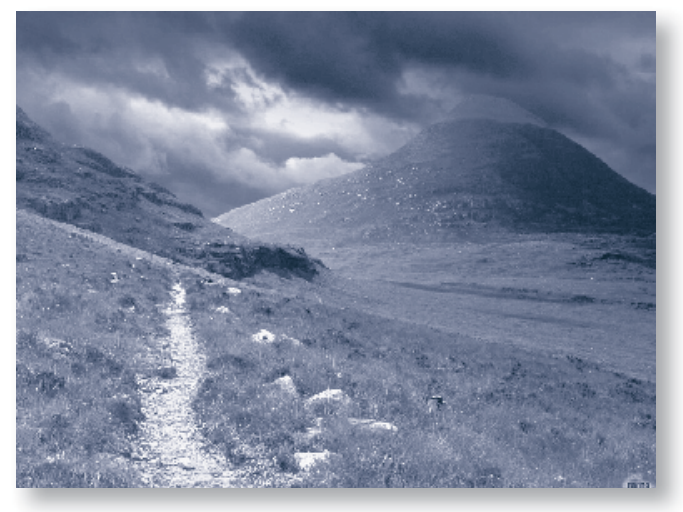

tencia sufrida, pero única y llena de significados.

Reflexionando sobre la enseñanza y la investigación, fortalecemos nuestra creencia de que la atención de enfermería requiere que los estudios no incluyan sólo los datos estadísticos, mientras estos sean esenciales para elucidar factores de riesgo, las ventajas terapéuticas, entre otros aspectos, pero creemos que la investigación cualitativa con abordaje fenomenológico puede proporcionar recursos para que sean aprendidas nuevas maneras de "mirar" a las personas que enferman, así como para la vida cotidiana de los lazos que se han establecido actualmente en la dinámica dentro y fuera del hospital.

\section{REFERENCIAS BIBLIOGRÁFICAS}

- ALVES, PC. (2006) A fenomenologia e as abordagens sistêmicas nos estudos sócio-antropológicos da doença: breve revisão crítica. Cad. Saúde Pública; 22(8): 15471554.

- DARTIGUES, A. (2003) O que é fenomenologia. São Paulo: Moraes.

- FREITAS, GF; MERIGHI, MAB; FERNANDES, MFP. (2007) La interface entre la fenomenología y el cuidado de enfermería. Index de Enfermería, España; 16(58): 55-8.

- GONÇALVES, R. (2005) Vivenciando o climatério: o corpo em seu percurso existencial à luz da fenomenologia [tese]. São Paulo: Escola de Enfermagem da USP.
- GOMES, AMDA; PAIVA, ES; VALDES, MTM. (2008) Fenomenologia, Humanização e Promoção da Saúde: uma proposta de articulação. Saúde Sociedade, São Paulo; 17(1): 143-52.

- HEIDEGGER, M. (2004) Ser e tempo. Trad. de Márcia Sá Cavalcante Schuback. 13a ed. Petrópolis: Vozes.

- JESUS, MCP; PEIXOTO, MRB; CUNHA, MHF. (1998) O paradigma hermenêutico como fundamentação das pesquisas etnográficas e fenomenológicas. Rev. LatinoAm. Enfermagem; 6(2): 29-35.

- LEITE, PC. (2006) A vivência de mulheres trabalhadoras de enfermagem que apresentam distúrbios osteomusculares Relacionados ao Trabalho (DORT): uma abordagem compreensiva da fenomenologia existencial. [tese]. São Paulo: Escola de Enfermagem da USP.

- LÓPEZ, L. (2010) Los Saberes Culturales en Salud en un Mundo Glocalizado. Index de Enfermería [Index Enferm] (edición digital); 19(2-3). Disponible en <http:// www.index-f.com/index-enfermeria/v19n2-3/2300. php> Consultado día 25 octubre 2010.

- MERIGHI,MAB. (2003) Abordagens teórico-metodológicas qualitativas: a vivência da mulher no período reprodutivo Rio de Janeiro: Guanabara Koogan.

- MOREIRA, DA. (2002) O método fenomenológico na pesquisa. São Paulo: Pioneira Thompsom.

- RIVERA, MS; HERRERA, LM. (2006) Fundamentos fenomenológicos para un cuidado comprensivo de enfermería Texto Contexto Enferm, Florianópolis; 15 (Especial): $158-63$.

- SILVA, JMO; LOPES, RLM; Diniz NM. (2008) Fenomenologia. Rev. bras. Enferm; 61(2): 254-257.

- TERRA, MG et al. (2006) Texto Contexto Enferm, Florianópolis; 15(4): 672-8. 